\title{
Eficacia de la creación de un programa de Escuela de pacientes en la detección de necesidades en pacientes con Poliquistosis Renal Autosómica Dominante
}

\author{
Helena García Llana ${ }^{1}$, Ramón Peces Serrano ${ }^{1}$, María Paz Ruiz-Álvarez ${ }^{1}$, María José SantanaValeros ${ }^{1}$, Ana \\ Isabel Castillo Plaza ${ }^{1}$, Carmen Parejo Fernández ${ }^{1}$, Filo Trocoli González ${ }^{1}$, Juan Carlos Julián Mauro², Emilio \\ López-Cuesta ${ }^{3}$, Rafael Selgas Gutiérrez ${ }^{1}$ \\ ${ }^{1}$ Servicio de Nefrología. Hospital Universitario La Paz-IdiPAZ. Madrid. España \\ ${ }^{2}$ Federación Nacional de Asociaciones para la Lucha Contra las Enfermedades del Riñón (ALCER) \\ ${ }^{3}$ Servicio de Radiodiagnóstico. Hospital Universitario La Paz-IdiPAZ. Madrid. España
}

Como citar este artículo:

García Llana H, Peces Serrano R, Ruiz-Álvarez MP, Santana Valeros MJ, Castillo Plaza AI, Parejo Fernández C, et al. Eficacia de la creación de un programa de Escuela de pacientes en la detección de necesidades en pacientes con Poliquistosis Renal Autosómica Dominante. Enferm Nefrol. 2019 Jul-Sep;22(3):293-301

\section{Resumen}

Introducción: La Poliquistosis Renal Autosómica Dominante es una enfermedad renal crónica responsable del $10 \%$ de los casos de insuficiencia renal terminal. La participación y los grupos de apoyo entre iguales son herramientas que mejoran el bienestar, evitando complicaciones y retrasando el avance de la enfermedad. Objetivos: Detectar necesidades informativas, así como recursos de apoyo, en este grupo de pacientes mediante la puesta en marcha de una Escuela de Pacientes con poliquistosis renal autosómica dominante.

Material y Método: Se utilizó un diseño mixto (cuantitativo y cualitativo). El estudio se desarrolló mediante cuatro fases: 1) Grupo focal: pacientes con poliquistosis renal y sus cuidadores; 2) Selección de los pacientes expertos; 3) Elaboración de los contenidos del programa de la Escuela de pacientes con poliquitstosis renal autosómica dominante; 4) Pilotaje del programa.

Resultados: Se detectaron necesidades de información referentes al tratamiento oral y al afrontamiento de la poliquistosis renal que no están cubiertas por los equipos de nefrología.

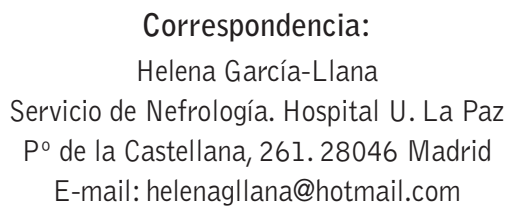

Conclusiones: La Escuela de Pacientes ha demostrado ser una herramienta útil para detectar necesidades y recursos en pacientes con poliquistosis renal autosómica dominante que han de enfrentarse a una enfermedad crónica donde se requiere la participación del paciente para garantizar la adhesión al tratamiento.

PALABRAS CLAVE: poliquistosis renal autosómica dominante; educación multidisciplinar; grupos de ayuda mutua.

Efficacy of the creation of a patient school program in the detection of needs in patients with autosomal dominant polycystic kidney disease

\section{Abstract}

Introduction: Autosomal Dominant Polycystic Kidney Disease is a chronic kidney disease responsible for $10 \%$ of cases of end-stage renal failure. Participation and peer support groups are tools that improve wellbeing, avoiding complications and delaying disease progression.

Objectives: To detect information needs, as well as support resources, in patients with autosomal dominant polycystic kidney disease trough a Patient School. 
Material and Method: A mixed design (quantitative and qualitative) was used. The study was developed through four phases: 1) Focus group: patients with autosomal dominant polycystic kidney disease and their caregivers; 2) Selection of expert patients; 3) Preparation of the contents of the program of the Patient School with autosomal dominant polycystic kidney disease; 4) Piloting the program.

Results: Information needs regarding oral treatment and coping with autosomal dominant polycystic kidney disease were detected, which are not covered by nephrology teams.

Conclusions: Patients School has proven to be a useful tool to detect needs and resources in patients with autosomal dominant polycystic kidney disease who have to face a chronic disease where patient participation is required to ensure adherence to treatment.

KEYWORDS: autosomal dominant polycystic kidney disease; multidisciplinary education; mutual aid groups.

\section{Introducción}

La poliquistosis renal autosómica dominante (PQRAD) es una enfermedad renal crónica que afecta a una de cada 500-1000 personas y que es responsable del 10\% de los casos de insuficiencia renal terminal (IRT) en España ${ }^{1}$. Es, a su vez, uno de los grupos de pacientes renales con mayores tasas de ansiedad y depresión, con las consecuencias que esto implica en cuanto a la falta de adhesión al tratamiento y el miedo a que otros miembros de la familia estén afectos ${ }^{2,3}$. Así mismo, este tipo de enfermedades renales hereditarias suponen un impacto tanto en la calidad de vida de los pacientes, como en la sostenibilidad de los sistemas sanitarios ${ }^{4}$. El desafío que supone adaptarse a una enfermedad crónica que implica tratamientos demandantes "de por vida" exige un esfuerzo por parte de los profesionales en desarrollar iniciativas comunitarias donde comparten escenarios educativos tanto profesionales como pacientes 5 . La evidencia describe que una relación clínica más simétrica, donde se tenga en cuenta la perspectiva del paciente genera mayor adhesión a tratamientos complejos ${ }^{6}$, así como satisfacción entre los profesionales sanitarios. Es por ello, que en la Escuela de Pacientes se van a promover dos ejes transversales educativos: promoción de los autocuidados y abordaje de los aspectos emocionales de vivir con una enferme- dad crónica. Por otro lado, estas acciones educativas quedan claramente justificadas si recordamos que en la línea estratégica $n^{\circ} 3$, del Documento Marco sobre Enfermedad Renal Crónica (ERC) dentro de la Estrategia de Abordaje de la Cronicidad en el Sistema Nacional de Salud (SNS) ${ }^{7}$, se destaca la importancia de incorporar mecanismos que fomenten la participación activa del paciente.

La Escuela de Pacientes trata de generar una alianza de trabajo compartido en la que tanto profesionales como pacientes dialoguen, desde posiciones de igualdad y promuevan los cambios necesarios para abordar los problemas que se presenten a lo largo del curso de la enfermedad. Su objetivo es ser un espacio de formación, encuentro e interacción bidireccional entre pacientes, asociaciones, familiares-cuidadores, profesionales sanitarios y ciudadanía en general, para intercambiar conocimientos y experiencias, con el objetivo de aportar las herramientas y habilidades que capaciten a todas las personas en su corresponsabilidad en el cuidado de su salud y control de la enfermedad. En España la única experiencia que tenemos en el ámbito de la PQRAD ha sido promovida a través de la Escuela Andaluza de Salud ${ }^{8}$. Nuestro grupo de trabajo lleva más de cinco años desarrollando acciones formativas para profesionales y pacientes dentro del ámbito de la $E R C^{9-12}$, pero todavía no se había diseñado ningún programa específico para la PQRAD.

Es por ello, que el objetivo del estudio fué detectar necesidades informativas, así como recursos de apoyo, en este grupo de pacientes mediante la puesta en marcha de una Escuela de Pacientes con PQRAD.

\section{Material y Método}

Se utilizó un diseño mixto (cuantitativo mediantes cuestionarios elaborados ad-hoc y cualitativo mediante la metodología de grupo focal).

\section{Principios del programa y setting}

Cada Escuela de Pacientes con PQRAD constaba de dos reuniones secuenciales intensivas tipo seminario.

- Primera sesión: metodología expositiva-participativa por parte de los profesionales sanitarios con espacio final para discusión y recogida de dudas. A esta primera sesión grupal acudieron 22 pacientes (15 pacientes y 7 familiares). 
- Segunda sesión: metodología activo-deliberativa con los profesionales, pacientes y sus familias. Mesa de discusión ajustada a las necesidades detectadas en el primer encuentro. La segunda sesión será liderada por los pacientes-expertos. A esta segunda sesión grupal acudieron 11 pacientes ( 7 pacientes y 4 familiares).

Los contenidos de la segunda sesión del programa han sido diseñados por los autores y el grupo de trabajo de atención integral a la PQRAD del Hospital Universitario La Paz (HULP). Las áreas temáticas educativas a trabajar estaban centradas en: avances en el tratamiento farmacológico, cuidados de enfermería en PQRAD, promoción de la adhesión al tratamiento, autocuidados, apoyo mutuo y afrontamiento activo de la enfermedad.

\section{Desarrollo de la Escuela de Pacientes con PQRAD}

\section{Fase 1: Reuniones de trabajo y grupo focal: pacientes con PQRAD y sus cuidadores}

Reuniones de trabajo: se llevaron a cabo cuatro reuniones interdisciplinares ( 2 nefrólogos, 1 psicólogo investigador, 2 pacientes) de cara a identificar las necesidades educativas.

Además de las reuniones de trabajo se realizó un grupo focal: $(n=7)$ facilitado por profesionales de la Escuela Andaluza de Pacientes de cara a detectar necesidades de información. Los asistentes fueron: 4 pacientes con $P R Q A D$, una cuidadora, un psicólogo investigador y un representante de la asociación de pacientes. El grupo de pacientes acudió en representación de toda España (Madrid, Pamplona, Cuenca y Alicante) y lo formaban dos hombres y dos mujeres. En la Tabla 1 pueden encontrarse los resultados del grupo focal sobre necesidades no cubiertas en la PQRAD.

Tabla 1. Necesidades de información identificadas por el focus group $(n=7)$.

Resultados del grupo focal

$\checkmark$ Avances en investigación sobre nuevos fármacos

$\checkmark$ Autogestión emocional

$\checkmark$ Estrategias de planificación familiar

$\checkmark$ Afrontamiento activo de la enfermedad

$\checkmark$ Impacto familiar

$\checkmark$ Autocuidados en PQRAD

$\checkmark$ Derechos laborales

$\checkmark$ Estilos de vida saludables en PQRAD

$\checkmark$ Alternativas TRS y apoyo en la toma de decisiones
Fase 2: Selección de los pacientes expertos: perfil Se llevó a cabo una revisión de la literatura para identificar los criterios de selección de pacientes expertos en el ámbito de la cronicidad. Se efectuaron búsquedas en las bases de datos MedLINE y PsycNFO, entre los meses de enero 2012 y diciembre 2017, utilizando los términos en inglés: "Autosomal dominant polycystic kidney disease", "Multidisciplinary Education", "Mutual support groups" y "Mentoring".

Los criterios extraídos de la literatura fueron:

- Ser enfermo o familiar renal.

- Acceder al programa voluntariamente.

- Tener capacidad de autocuidado.

- Tener un estilo de afrontamiento activo de la enfermedad.

- Ausencia de discapacidades psíquicas.

- Mostrar empatía, interés por ayudar y motivación.

- Poseer habilidades de comunicación y conducción del grupo.

La psicóloga del servicio de nefrología realizó una breve entrevista telefónica de cara a garantizar que se cumplían los criterios de inclusión en el programa. Los pacientes expertos fueron citados a una reunión liderada por el jefe del servicio, donde se explicaron los objetivos y principios del programa.

\section{Fase 3: Elaboración de los contenidos del programa} de la Escuela de pacientes con PQRAD

El programa se diseñó en dos sesiones, una expositiva, liderada por ALCER Madrid y conducida principalmente por profesionales médicos y otra activo-participativa, liderada por pacientes, enfermería y psicología. La duración final del programa fue de 7 horas.

\section{Fase 4: Pilotaje del programa}

En la sección de resultados se presentan los datos cuantitativos y cualitativos extraídos de esta primera experiencia.

\section{Medidas y variables}

Las medidas fueron aplicadas después de cada una de las sesiones educativas. Se administraron cuestionarios tipo encuesta de cara a identificar el perfil clínico, así como las necesidades de información de los asistentes. Todos los instrumentos fueron elaborados ad-hoc. También se incluyó una medida de satisfacción con formato de respuesta tipo escala Likert de 5 puntos ( $1=$ puntuación más baja; $5=$ puntuación más alta). Ver Anexos. 


\section{Análisis estadísticos}

Se realizaron análisis descriptivos que incluyeron: medias, desviaciones típicas y rangos para las variables cuantitativas, y tablas de frecuencias para las variables cualitativas. Los datos fueron analizados con el programa informático SPSS versión 22.0.

\section{Garantías éticas}

El estudio cumple con todas las garantías éticas necesarias para su desarrollo. No se han incluido datos de carácter personal que pudieran identificar a los pacientes de forma directa o indirecta, respetando los principios éticos y universales, así como, las normas internacionales de protección de datos y la legislación española vigente.

\section{Resultados}

Primera sesión: "Jornada informativa para pacientes y familiares con PQRAD de ALCER Madrid: Hablemos de PQRAD"

\section{Perfil de los asistentes}

Se recogieron un total de 22 encuestas ( 15 pacientes y 7 familiares), lo que supuso un $63 \%$ de la muestra total que acudió a las Jornadas Informativas de PQRAD organizadas por ALCER Madrid ( $n=35)$. La edad media de los asistentes fue de 53 años (DE:9,081). El $59 \%$ eran mujeres. El $90 \%$ de los asistentes eran españoles, con presencian de dos sujetos de otras nacionalidades. El $82 \%$ estaba casado/vivía en pareja y un $55 \%$ estaba con ocupación laboral activa.

\section{Avances en el tratamiento y necesidades de informa- ción \\ El tratamiento farmacológico, de reciente incorporación al mercado, es conocido por el $73,3 \%$ de la muestra. No así sus efectos secundarios, que solo son conocidos por el $63,3 \%$. Un $36 \%$ de la muestra refiere no haber teni- do oportunidad de discutir este nuevo tratamiento con su nefrólogo de referencia. El 100\% de este sub-grupo desearía poder hacerlo.}

La Tabla 2 recoge las cinco principales preocupaciones sobre el tratamiento farmacológico que refiere la muestra encuestada.

Por último en la Tabla 3 se recogen las principales necesidades de información que demanda la muestra encuestada de cara a futuras ediciones.
Tabla 2. Cinco principales preocupaciones relacionadas con el tratamiento farmacológico $(n=22)$.

\begin{tabular}{l|l}
\hline $\begin{array}{l}\text { Principales preocupaciones relacionadas } \\
\text { con el tratamiento farmacológico }\end{array}$ & $\begin{array}{l}\text { Datos } \\
\text { N }(\%)\end{array}$ \\
\hline Dosis tolerable & $8(36,6)$ \\
\hline Efectividad del tratamiento & $7(31,8)$ \\
\hline Adhesión al tratamiento "de por vida" & $4(18,1)$ \\
\hline Complicaciones hepáticas & $2(9,0)$ \\
\hline Acceso equitativo al tratamiento & $1(4,5)$ \\
\hline
\end{tabular}

Tabla 3. Necesidades de información $(n=22)$.

\begin{tabular}{|l|l|}
\hline $\begin{array}{l}\text { Necesidades de información } \\
\text { Sobre la PQRAD }\end{array}$ & $\begin{array}{l}\text { Datos } \\
\text { N }(\%)\end{array}$ \\
\hline Afrontamiento de la PQRAD & $7(31,8)$ \\
\hline Avances en investigación & $4(18,2)$ \\
\hline Autocuidados para frenar progresión & $3(13,6)$ \\
\hline Descendencia e impacto familiar & $3(13,6)$ \\
\hline TDC e inicio TRS & $3(13,6)$ \\
\hline Otras & $2(9,1)$ \\
\hline TDC = toma de decisiones compartida; TRS = tratamiento renal sustitutivo.
\end{tabular}

Segunda sesión: "Escuela de Pacientes con PQRAD: Tanto si crees que puedes, como si crees que no puedes, estás en lo cierto"

\section{Perfil de los asistentes}

Se recogieron un total de 11 encuestas ( 7 pacientes y 4 familiares). Todos los participantes habían asistido a la primera sesión. La edad media de los asistentes fue de 57 años (DE:9,370). El 63\% eran mujeres. El 91\% de los asistentes eran españoles, con presencian de un sujeto de otra nacionalidad. El $72,7 \%$ estaba casado/ vivía en pareja y solo un $45,5 \%$ estaba con ocupación laboral activa. El $45 \%$ de los participantes $(n=5)$ es tratado en un centro sanitario diferente al HULP.

\section{Satisfacción de los asistentes}

La satisfacción de los asistentes fue alta. Ver Tabla 4. Todos los participantes (11/11) recomendarían este tipo de iniciativas a otras pacientes/familiares en sus circunstancias. Así mismo, se dio la oportunidad de añadir sus propios comentarios; algunos incluyeron observaciones del tipo "Esta línea de trabajo es muy necesaria y este tipo de escuela debería repetirse todos los meses". 
Tabla 4. Satisfacción de los participantes de la segunda sesión $(n=11)$.

\begin{tabular}{|l|c|}
\hline Componente & Media $^{1}$ \\
\hline Importancia de la escuela & 4,91 \\
\hline Relevancia del contenido & 4,91 \\
\hline Utilidad de la metodología & 4,82 \\
\hline Ambiente entre los participantes & 5,00 \\
\hline Satisfacción con los profesores & 4,91 \\
\hline Satisfacción global & 4,91 \\
\hline
\end{tabular}

${ }^{1}$ Escala Likert donde 5 es la máxima puntuación.

\section{Discusión}

Uno de los principales hallazgos de este estudio fue que la participación de los pacientes y familiares en una Escuela de Pacientes ha resultado altamente satisfactoria para todos los agentes implicados. Así mismo se ha podido realizar una detección de necesidades que nos ayudará a diseñar espacios educativos futuros. Las principales preocupaciones de los pacientes y sus familiares giraban en torno a tres ejes: afrontamiento de la enfermedad, avances en investigación, autocuidados e impacto familiar. Esto coincide con estudios previos de ámbito más genérico de la enfermedad renal ${ }^{13,14}$. Cada vez más autores nos recuerdan la importancia de tener en cuenta la perspectiva del paciente ${ }^{15}$, ya que permite a los clínicos diseñar programas ajustados a las necesidades de los pacientes.

Otras comunidades autónomas han elaborado programas más ambiciosos y dotados de más recursos dentro del ámbito enfermedad renal crónica que nos han servido de inspiración para el diseño de nuestra Escuela ${ }^{16-17}$. Si bien estos programas son más genéricos dentro del ámbito de la enfermedad renal crónica y no recogen las peculiaridades de la PQRAD.

Este programa describe preocupaciones de los pacientes relacionadas con la promoción de salud en relación con las dosis tolerables y la efectividad de tratamiento oral. Nuestra experiencia coincide con la de otros programas peer-to-peer implementados en otro tipo de condiciones crónicas de salud como la hipertensión ${ }^{18} 0$ la epilepsia ${ }^{19}$.

Cuando se habla de procesos crónicos de enfermedad se tiene en cuenta al paciente y a la familia como unidad a la que atender. Desde un hospital de agudos, diseñado para centrarse monográficamente en las enfermedades y en los pacientes, la implementación de una Escuela de Pacientes ayuda a incorporar a la familia y a la comunidad como foco atencional al promover la sensibilidad en los profesionales frente a la importancia de los de autocuidados en una enfermedad de larga evolución. Desde esta visión, se explicita la necesidad de abordar de forma integrada las patologías y la experiencia de sufrimiento o, dicho de otro modo, la perspectiva biológica y la biográfica de los pacientes.

Si deseamos proporcionar una adecuada atención a los pacientes con PQRAD se requiere un abordaje biopsicosocial e interdisciplinar, centrado en el paciente, que integre la multidimensionalidad del ser humano y donde tengan lugar espacios de apoyo entre iguales ${ }^{20,21}$.

Este programa no está exento de limitaciones. En primer lugar, no se trataron todos los temas detectados en la primera sesión por falta de tiempo y cuestiones organizativas. Es por ello que la continuidad de la Escuela se impone como una realidad dentro del Servicio, y porque así los pacientes nos lo han solicitado.

A partir de los resultados obtenidos podemos observar que la Escuela de Pacientes ha demostrado ser una herramienta útil para detectar necesidades y recursos en pacientes con $P Q R A D$ que han de enfrentarse a una enfermedad crónica donde se requiere la participación del paciente para garantizar la adhesión al tratamiento. Este modelo de intervención solo puede hacerse realidad si logramos incorporar a los propios pacientes como agentes de cambio y les damos voz dentro de los espacios colectivos de cuidado y promoción de salud.

\section{Agradecimientos}

Cátedra de patrocinio OSTUKA. Fundación Universidad Autónoma de Madrid.

\section{Conflicto de intereses}

Los autores no presentan ningún conflicto de interés. 
Recibido: 20-01-19

Revisado: 01-02-19

Modificado: 25-03-19

Aceptado: 01-04-19

\section{Bibliografía}

1. Alianza frente a la PQRAD. Libro blanco de la PQRAD en España. Madrid. Cícero Comunicación, 2016.

2. Pérez-Domínguez TS, Rodríguez-Pérez $A$, Buset-Ríos N, Rodríguez-Esparragón $F_{\text {, Gar- }}$ cía-Bello MA, Pérez-Borges $P$, Parodis-López $Y$, Rodríguez-Pérez JC, en nombre del Grupo de Investigación Hiricare. Psiconefrología: Aspectos psicológicos en poliquistosis renal autosómica dominante. Nefrología. 2011;31(6):716-22.

3. Simms RJ, Thong KM, Dworschack GC, Ong ACM. Increased psychosocial risk, depression and reduced quality of life living with autosomal dominant polycystic kidney disease. Nephrology, Dialysis and Transplantation. 2016;31:1130-40.

4. Devuyst 0, Knoers NV, Remuzzi G, Schaefer F. Rare inherited kidney diseases: challenges, opportunities, and perspectives. Lancet. 2014 May 24:383(9931),1844-59.

5. Hughes J, Wood E, Smith G. Exploring kidney patients' experiences of receiving individual peer support. Health Expect. Dec 2009;12(4):396-406.

6. Faulk JS. Peer-to-peer transplant mentor program: the San Diego experience. Transplant Proc. Jun 1999;31(4A):75S.

7. Documento Marco sobre Enfermedad Renal Crónica dentro de la Estrategia de Abordaje de la Cronicidad en el Sistema Nacional de Salud. Madrid. Centro de Publicaciones del Ministerio de Sanidad, Servicios Sociales e Igualdad. 2015.

8. Escuela Andaluza de Salud Pública. Escuela de Pacientes. Poliquistosis Renal Autosómica Dominante. [Consultado 8 abril 2019]. Disponible en: https://www.easp.es/virtual/docencia/course/view. php?id=1039.

9. García-Llana H, Barbero J, Remor E, Díaz-Sayas L, Rodríguez-Rey R, del Peso G, Selgas, R. Impacto de un curso interdisciplinar de formación en Counselling y apoyo en la toma de decisiones a profesionales de un Servicio de Nefrología. Nefrología. 2011; $31(3): 322-30$.

10. García-Llana H, Barbero Gutiérrez J, Remor Bitencourt E, Celadilla Díez 0, Trocoli González 0, del Peso Gilsanz G, Selgas Gutiérrez, R. Beneficio de la realización de un taller en gestión emocional para enfermería nefrológica. Enfermería Nefrológica. 2012;15(3):176-81. [Consultado 8 abril 2019]. Disponible en: http://www.revistaseden.org/ files/3137_3.pdf.

11. García-Llana H, Rodríguez-Rey R, Selgas, R. Formación en asesoramiento psicológico (Counselling) y apoyo emocional a residentes de Nefrología: Estudio Piloto. Sociedad Iberoamericana de Información Científica (SIIC). 2014;20:362-7. Sección On-line: Columnista Experto.

12. García-Llana H, Bajo MA, Barbero J, del Peso G, Selgas, R.. The Communication and Bioethical Training ( $\mathrm{CoBiT}$ ) Program for assisting dialysis decision-making in Spanish ACKD Units. Psychology, Health \& Medicine. 2017;22(4):474-82.

13. Schipper K, Abma TA. Coping, family and mastering: Top priorities for social science research by patients with chronic kidney disease. Nephrol Dial Trasplant. 2011;26(10):3189-95.

14. Tong A, Rangan GK, Ruospo M, Saglimbene V, Strippoli GFM, Palmer SC, Tunnicliffe DJ \& Craig JC. A painful inheritance-patient perspectives on living with polycystic kidney disease: thematic synthesis of qualitative research. Nephrology, Dialysis and Transplantation. 2015;30:790-800.

15. Mayer M. Seeking what matters: patients as research partners. The Patient. 2012;5(2):71-4.

16. Escuela de pacientes con Enfermedad Renal Crónica. Rioja Salud. [Consultado 8 abril 2019]. Disponible en: https://escuelapacientes.riojasalud.es/.

17. Aguilera Flórez AI, Prieto Velasco M, González Romero L, Abad Toral B, Martínez Crespo E, Robles del Rio I, Gutiérrez Gutiérrez E, Calleja Fernán$\operatorname{dez} A$, de Boso Serrano P. Una estrategia poco uti- 
lizada en el cuidado de pacientes con Enfermedad Renal Crónica: la educación en grupo y multidisciplinar de pacientes y sus familiares. EnfermeríaNefrológica.2012;15(1):14-21. [Consultado 8 abril 2019]. Disponible en: http://www.revistaseden.org/ files/3083_unaestrategia.pdf.

18. Whittle J, Schapira MM, Fletcher KE, Hayes $A$, Morzinski J, Laud P, Eastwood D, Ertl K, Patterson $L$, Mosack KE. A randomized trial of peer-delieverd self-management support for hypertension. American Journal of Hypertension. 2014;27(11):141622.
19. Elafros MA, Mulenga J, Mbewe $E$, Haworth $A$ Chomba E, Atadzhanov M, Birbeck GL. Peer suport groups as an intervention to decrease epilepsy associated stigma. Epilepsy Behav. 2013;27(1):188-92.

20. Brunier G, Graydon J, Rothman B, Sherman C, Liadsky R. The psychological well-being of renal peer support volunteers. J Adv Nurs. Apr. 2002:38(1):40-9.

21. Borrell F. El modelo biopsicosocial en evolución. Medicina Clínica. 2002;119(5):175-9. 